\title{
LA TUBERCulose des POISSONS
}

\author{
par P. BESSE \\ Docteur véterinaire \\ Chargé du Laboraloire de Pathologic Pisciaire.
}

Il est généralement admis que les animaux à sang froid et les poissons en particulier sont réfractaires à la tuberculose, car ils ne contractent pas d'infection tuberculeuse lorsqu'on leur injecte des bacilles de la tuberculose humaine, bovine ou aviaire. Sur ce point, d'ailleurs, les auteurs ne sont pas parfaitement d'accord et des expériences à longue échéance sont en cours pour vérifier cette assertion.

Est-ce à dire que les poissons soient plus favorisés que les animaux supérieurs? Il n'en est rien.

En effet, une affection tuberculeuse pisciaire a été décrite par quelques auteurs, mais jusqu'à ces derniers temps semblait devoir être exceptionnelle.

C'est en 1897 que de Dubard, Bataillon et Terre signalèrent pour la première fois ce nouveau type de tuberculose sur une Carpe (Cyprinus carpio), provenant d'une pisciculture à Velars-sur-Ouche (Côte-d'Or). Cet animal présentait, sur le côté droit de l'abdomen une tumeur ve la grosseur d'un œuf de pigeon logée entre l'ovaire et la paroi musculaire et contenant une pulpe non caséeuse. Cette pulpe contenait une grande quantité de bacilles présentant des caractères morphologiques et tinctoriaux très voisins de ceux du bacille de la tuberculose humaine. Il put être isolé et cultivé et se montra alors différent de son illustre parent ; il a été dénommé Bacillus tuberculosis piscium.

C'est surtout grâce à un caractère tinctorial particulier que l'on différencie les germes de la famille des Mycobactéries et, parmi eux, les bacilles tuberculeux de tous les autres microbes.

Les Mycobactéries se colorent fortement en rouge par la fuchsine phéniquée à chaud et ne se décolorent plus sous l'action d'un acide fort dilué tel que l'acide nitrique au $1 / 3$ ou l'acide sulfurique au $1 / 10^{e}$. Les autres bactéries se décolorent; on les recolore ensuite au bleu de méthylène; c'est la méthode de Ziehl ; on dit que les Mycobactéries sont acido-résistantes. Sur une préparation, elles se distinguent fortement en rouge sur le fond bleu; les autres bactéries sont bleues.

Après de Dubard, Bataillon et Terre, Bartarelli et Bocchia, 
en 1910, annoncent que la tuberculose spontanée sur les poissons vendus sur les marchés est rare.

En 1913, Alexander trouve un ulcère tuberculeux de la Morue (Gadus callarias).

Sutherland, en 1922, signale des Mycobactéries dans le tissu souscutané et les organes du Halibut (Hyppoglossus hyppoglossus).

- Aronson, en 1926, décrit une enzootie tuberculeuse dans deux bacs de l'aquarium marin de Philadelphie; dans l'un se trouvent des "Sergeants Major " (Abudefduf mauritinus), dans l'autre des "Micropognon".

En 1941, JaHNel fait savoir qu'une maladie tuberculeuse a lieu très souvent chez les poissons d'aquarium à eau chaude, surtout sur les Danio, Gymnocorymbus et IIyphessobrycon.

Ayant été hébergé très obligeamment à l'aquarium du Musée de la France d'Outre-Mer, de Janvier 1947 à Avril 1949, afin d'étudier la pathologie pisciaire, il m'a été donné d'observer de très nombreux cas de tuberculose chez les poissons exotiques vivant en aquarium en eau chaude.

C'est ainsi que, sur 288 poissons morts de maladie microbienne ou de maladie à protozoaires, j'ai trouvé 243 fois une affection à bacilles acidorésistants, soit plus de $80 \%$. D'ailleurs, je retrouve cette même proportion dans la mortalité que j'ai pu constater soit dans les bacs des éleveurs de poissons exotiques, soit dans les aquariums des particuliers.

Ces affections à bacilles acido-résistants se produisent généralement sous la forme d'une épizootie lente dans un aquarium. Parfois, cependant, nous n'avons observé qu'un cas sur toute la population d'un bac. Certaines familles semblent être particulièrement sensibles à l'action de ces micro-organismes ; c'est ainsi que, proportionnellement à leur population, les Anabantidés sont très fortement atteints avec 101 morts ainsi que les Characinidés : 32 morts, les Mélanotaenidés : 7 unités (la totalité), les Cyprinodontidés : 32 (la presque totalité). Puis viennent les Cyprinidés exotiques : 40 morts, les Poecilidés : 24, ce qui est assez peu, les Cichlidés : 4 , ce qui est rare, et les Centrarchidés : 3 .

Dans ces familles, certaines espèces sont très sensibles et d'autres ne paient aucun tribut; ainsi, dans les Characinidés, Hyphessobrycon flameus est très souvent atteint, alors que nous n'avons jamais observé la maladie chez Pristella ridlei.

D'autre part, certaines espèces présentent de préférence des formes particulières de l'affection. Nous allons les examiner séparément. Nous les avons dénommées :

la Tuberculose des Macropodes,

l'Ascite des combattants,

l'Exophtalmie des Danios,

la Maladie caséeuse des Cyprins,

le Marasme des Poecilidés. 


\section{I. - LA TUBERCULOSE DES MACROPODES}

Symptômes. - Les poissons malades présentent des lésions cutanées, les écailles se hérissent et tombent à l'emplacement des lésions, l'épiderme et le derme présentent une solution de continuité et la lésion apparaît rouge, congestionnée; il n'est pas remarqué d'épanchements sanguins.

Parfois un œil est le siège d'une kérato-conjonctivite, puis d'un glaucome et fait saillie hors de l'orbite. Le poisson borgne ou aveugle présente alors des noircissements de tout ou partie du corps.

Sur certains sujets, après un laps de temps variant de huit jours à un mois après le début de l'apparition des lésions, on remarque une dilatation progressive de la cavité abdominale; en même temps apparaît le " hérissement des écailles ".

Chez d'autres sujets, au contraire, on constate un amaigrissement progressif allant jusqu'à la cachexie.

La mort survient après une évolution plus ou moins longue de la maladie (de quelques jours à quelques mois).

Autopsie. - Chez tous les poissons on observe des lésions très étendues des reins; ils paraissent gonflés, blanchâtres et contiennent toujours des masses caséeuses jaunâtres, variant de la grosseur d'une petite tête d'épingle à celle d'un pois chiche.

Le foie est jaunâtre, bistre; il présente également des lésions caséeuses.

Chez les sujets hydropiques, l'abdomen contient un liquide clair, incolore, légèrement opalescent.

Examen microscopique. - Sur des frottis de reins, de foie, de lésions, après coloration par la méthode de Gram, on trouve en très grande abondance un microbe Gram positif se colorant irrégulièrement et très difficilement; il présente de nombreuses granulations violettes et le reste du corps microbien est à peine visible.

Les bacilles se trouvent soit en masse compacte, serrés les uns contre les autres, en bottes ou en pelote d'épingles, soit isolés. Il s'agit d'un microbe droit ou légèrement incurvé à bout arrondi de $1,5 \mu$ sur $0,2 \mu$, il est immobile et non sporulé.

Coloration de ZIEHL-NIELSSEN : ce microbe est acido alcoolo-résistant ; il est coloré en rouge et les granulations apparaissent en rouge plus vif.

Ensemencements. - Des ensemencements ont été faits à partir du rein, du foie, du liquide d'ascite, des lésions sur les milieux les plus divers : gélose inclinée, gélose glycérinée, gélose à l'extrait globulaire, gélosesérum, gélose au sang; en milieu liquide dans bouillon nutritif, bouillon glycériné, bouillon-sérum, bouillon-extrait globulaire, bouillon-ascite; sur milieux spéciaux : sérum coagulé, glucose peptoné (milieu de Loêffler), milieux de Lœwenstein, de Petragnani, de Jensen et Jensen glycériné, milieux de Laporte et de Besredka. 
Des ensemencements en milieu anaérobie sur gélose Veillon, gélose gélatine, bouillon V. F. contenant des fragments d'organes : foie, reins, fibrine, albumine d'œufs, furent faits.

Voici les résultats obtenus :

$1^{\circ}$ aucune culture sur les milieux anaérobies. Ce bacille est donc aérobie strict;

$2^{\circ}$ sur les autres milieux, je n'ai obtenu aucune culture sauf sur les milieux de Loewenstein, Petragnani, Laporte, Jensen, Jensen glycériné, sérum coagulé et Loëffler.

Le bacille cultive à une température variant de 12 à $33^{\circ}$, l'optimum de température étant aux environs de 25\%. Aux températures de 12 à $33^{\circ}$ la culture est très lente et très faible.

Le microbe ne cultive pas à la température de $37^{\circ}$; cette température est même léthale, puisque des tubes placés pendant quarante-huit heures dans une étuve à $37^{\circ}$ et reportés à $25^{\circ}$ ne présentent jamais de prolifération.

Aspect des cultures. - Sur le milieu classique de Loewenstein à 250, on voit apparaître au bout de sept à huit jours, à peine visibles à l'œil $\mathrm{nu}$, de très petites colonies qui, au bout de quinze jours, peuvent atteindre $1 / 2$ millimètre et 1 millimètre en vingt et un jours. Nous pouvons donc considérer que le bacille cultive en quinze jours, trois semaines.

Les cultures âgées d'un mois et plus prennent un aspect granuleux mamelonné ; très légèrement lisses, elles sont d'une couleur allant du crème au jaune légèrement orangé.

Ces cultures sont assez adhérentes au milieu et ont la consistance des pains de levure de boulangerie.

Mêmes caractères culturaux sur les milieux de Jensen glycériné et de Petragnani, avec cette différence que la croissance est plus abondante sur le milieu de Jensen et sur le milieu de Petragnani; sur ce dernier la croissance est même légèrement plus rapide (dix à quinze jours).

La vitesse de croissance est également de trois semaines environ sur sérum coagulé, milieu de Laporte et milieu de Loëffler, mais l'aspect des cultures est différent. Les colonies peuvent atteindre une dimension de 1 à 2 millimètres; elles sont rondes, bombées, luisantes, d'une couleur variant du blanc au jaune orangé; lorsqu'elles sont coalescentes, elles forment un enduit crémeux, luisant, jaune sale très pâle et s'émulsionnent facilement.

J'ai réussi, assez difficilement d'ailleurs, à adapter le microbe à la pomme de terre glycérinée ; la première culture de ce milieu a mis sept mois à pousser. A l'heure actuelle, à la température de $25^{\circ}$, la culture pousse en trois semaines environ; c'est une culture en chou-fleur identique à celle du bacille tuberculeux humain, de couleur café au lait un peu foncé ; un voile épais ridé se développe dans le liquide contenu dans le tube.

J'ai, parfois, également obtenu un début de culture sur gélose à la 
pomme de terre et sur un mélange à parties égales de bouillon nutritif et de sérum humain.

Le microbe cultive bien dans le milieu de Dubos; Il a été dénommé Mycobacterium anabanti.

\author{
II. - L'ASCITE DES COMBATTANTS \\ (Betta splendens, famille des Anabantidés).
}

Environ $50 \%$ des combattants que j'ai observés meurent de la même façon dès qu'ils atteignent 1 an et demi à 2 ans. On voit l'abdomen augmenter progressivement de volume, les écailles se séparent, la peau se distend et, au bout d'un laps de temps de un à deux mois, cette peau devient transparente et le ventre atteint la dimension d'une grosse noisette ou d'une petite noix.

Les mouvements deviennent de plus en plus gênés, les déplacements sont de plus en plus courts et pénibles. L'animal reste dans un coin de l'aquarium, posé sur le fond, puis ses déplacements deviennent désordonnés. Il se nourrit encore ; cependant ce n'est qu'à la période préagonique que, perdant tout contrôle, le combattant nage sur le côté dans le fond de l'aquarium, ou le ventre en l'air près de la surface. Arrivé à ce stade, la mort survient en général dans les vingt-quatre heures.

Si l'on ponctionne, on recueille un liquide clair, limpide, légèrement visqueux, se coagulant partiellement. Après étalement et coloration on trouve peu d'éléments figurés, quelques rares lymphocytes et parfois un ou deux bacilles acido-résistants. Les organes abdominaux sont fortement refoulés vers la périphérie. Parfois, le rein et le foie présentent de petits nodules caséeux. Ces nodules étalés et colorés se montrent chargés d'un plus ou moins grand nombre de bacilles acido-résistants.

Ces bacilles sont petits, grêles, pointus ou arrondis aux deux extrémités de $1 \mu$ à $1,2 \mu \times 0,10 \mu$ à $0,15 \mu$. Ils sont uniformément colorés, prennent le Gram et restent colorés par la méthode de Ziehl-Nielsen. $\mathrm{J}$ 'ai pu isoler une souche de ce microbe; elle se présente sous la forme de colonies hémisphériques crémeuses, luisantes, jaune d'or, cultivant en quelques jours sur le milieu de Loewenstein.

Il ne m'a pas toujours été possible de mettre en évidence la présence d'une mycobactérie.

Les mêmes symptômes ont été observés chez un Danio albolinatus, famille des Cyprinidés.

\title{
III. - L'EXOPHTALMIE DES DANIO MALABARICUS (Ciprinidés)
}

Fin mars 1948, notre attention fut attirée sur cette curieuse affection sur des couples que nous avions mis en reproduction : les poissons semblaient bien portants et la pariade avait lieu normalement. Un matin, 
nous fûmes surpris de trouver une femelle avec un œil en moins, le lendemain l'autre œil avait également disparu. Nous avons tout d'abord pensé à une mutilation consécutive à un combat.

La chose se reproduisit sur deux ou trois autres femelles, mais le fait nous sembla étrange lorsque les mâles qui restaient alors seuls dans leurs aquariums présentèrent les mêmes symptômes.

La maladie se manifeste sur les animaux de deux à trois ans, au moment de la période de frai ; le ventre est augmenté de volume, mais ce phénomène passe généralement inaperçu, les poissons ayant normalement le ventre dilaté avant la ponte.

Les couleurs restent vives.

Progressivement; en une quinzaine de jours, les yeux (parfois un seul œil) font de plus en plus saillie en dehors des orbites, le globe oculaire est légèrement augmenté de volume, puis un oil se détache (je n'ai pu observer par quel mécanisme). L'animal ne semble pas être très affecté par cette mutilation.

De un à trois jours après, le deuxième œil se détache à son tour. Le poisson, aveugle, complètement désorienté, ne s'alimente plus et meurt en quelques jours.

A l'autopsie, j'ai trouvé le foie et le rein très augmentés de volume, bourrés de petites granulations caséeuses blanc-jaunâtre, de la grosseur d'une tête d'épingle. Parfois, j'ai observé de petits chancres sur les branchies.

Des colorations par la méthode de Ziehl-Nielsen sur des frottis de foie et de rein montrent la présence d'une quantité extraordinaire de bacilles acido-résistants; nous avons retrouvé les mêmes bacilles sur les lésions nécrotiques des branchies.

Derrière les globes oculaires exorbités, nous avons noté la présence d'une masse épaisse crémeuse, blanche, qui provoque la saillie de l'œil ; cette masse s'est avérée être une culture pure du bacille trouvé dans les lésions internes.

Le microbe est un bâtonnet droit ou légèrement incurvé, pointu ou arrondi à ses deux extrémités, mesurant de $1,5 \mu$ à $3,5 \mu \times 0,2 \mu$ à $0,5 \mu$; il se colore uniformément, est parfois granuleux ou barré et prend le Gram; il reste coloré par la méthode de Ziehl.

J'ai pu l'obtenir en culture pure. La colonie est hémisphérique, luisante, crémeuse, jaune d'or, semblable à celles que nous avons isolées d'autres espèces de poissons (Gymnocorynbus, Rivulus, Lebistes, Melanotaenia, Combattant, etc.).

\section{IV. - MALADIE CASÉEUSE DES CYPRINS}

Cette affection, constatée pour la première fois sur le poisson rouge (Carassius auratus) dans sa variété "poisson voile " ou " poisson japonais", a été également observée chez un cyprinidé exotique : Barbus conchonius et chez un Anabantidé : Trichopodus trichopterus ou "Gourami ". 
Seuls les vieux sujets sont atteints; l'affection se manifeste par une sorte de bubon qui se développe lentement sur la paroi abdominale et généralement à la partie antéro-supérieure gauche dans la région située en arrière du cœur. Cette proéminence de forme conique à pointe mousse ne présente aucun caractère inflammatoire et ne crève pas spontanément comme un abcès. D'autres saillies semblables peuvent se produire en d'autres parties de l'abdomen. L'animal atteint peut vivre très longtemps sans que son existence semble autrement troublée; la mort survient sans symptôme particulier. A l'autopsie, on trouve à l'emplacement de la saillie cutanée une masse caséeuse volumineuse lobée qui repousse simplement la peau. Dans tout l'abdomen, sur tous les organes, dans le foie, dans les reins et la rate, on trouve une quantité extraordinaire de masses caséeuses de la grosseur d'un grain de blé à celle d'un pois, tantôt seules, tantôt agglomérées en masses volumineuses ; elles intéressent le péritoine, le mésentère, les tissus hépatique, rénal, splénique. Ces masses sont amorphes et ont la consistance du suif. Étalées et colorées par la méthode de Ziehl, il m'a été rarement permis d'y mettre en évidence un bacille acido-résistant. Sur un très grand nombre de lames et après des recherches systématiques, on trouve un ou deux bacilles. Je n'ai pu les obtenir en culture.

Chez Trichopodus trichopterus les bacilles sont beaucoup plus nombreux et on les trouve en gros amas dispersés.

Il faut rapprocher de cette affection la maladie épizootique constatée chez un Characinidé : Hyphessobrycon flameus et chez un Anabantidé : Ctenops vittalus. Le ventre est également bosselé, augmenté de volume et contient de très nombreuses et volumineuses masses caséeuses; les viscères en sont de même truffés.

Ce qui différencie ces affections de la maladie caséeuse, c'est la présence d'une quantité extraordinaire de bacilles acido-résistants qui se présentent en amas volumineux. Ce sont des bacilles droits, aux extrémités arrondies, se colorant uniformément, de $1 \mu \times 0,10 \mu$. J'ai observé plusieurs cultures sur milieu de Loewenstein. Ce sont des colonies blanches, crémeuses, lisses ou légèrement granuleuses.

\section{V. - LE MARASME DES POECILIDÉS}

Cette affection a été observée sur Xiphophorus helleri, Platypoecilus maculatus, Lebistes reticulatus (Guppy), Mollienisia; dans toutes leurs variétés.

L'animal atteint maigrit progressivement, son dos se vousse, son ventre se rentre, les yeux se creusent, les arêtes deviennent apparentes sous la peau et les écailles. Il ne mange plus et reste isolé dans un coin de l'aquarium où il est l'objet d'attaques continuelles de ses congénères. Au bout d'un laps de temps qui dépasse rarement un mois, il meurt.

A l'autopsie, on trouve les viscères d'apparence normale. Quelquefois, on aperçoit de nombreuses petites granulations jaunâtres sur le foie et les reins. Des frottis de ces organes colorés par la.méthode de 
Ziehl montrent la présence de nombreux bacilles acido-résistants disséminés ou en amas.

J'ai pu obtenir une culture de ces bacilles : colonies hémisphériques, jaune d'or, crémeuses, luisantes.

La morphologie et l'aspect cultural sur Loewenstein sont identiques à ceux décrits chez Danio malabaricus.

Liste des poissons trouvés porteurs à l'autopsie de lésions à bacilles acido-résistants.

\begin{tabular}{|c|c|c|}
\hline FAMILLES & ESPECES & NOMBRE \\
\hline ANABANTIDAE $\ldots \ldots \ldots$ & 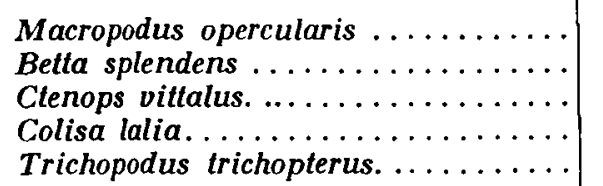 & $\begin{array}{r}52 * \\
18 * \\
13 * \\
5 \\
13\end{array}$ \\
\hline Characinidae. . . . . . & 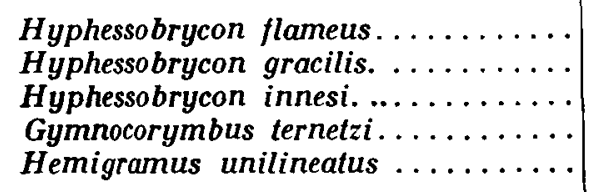 & $\begin{array}{r}15 * \\
1 \\
4 \\
8 * \\
4 *\end{array}$ \\
\hline CyPRINODONTIDAE. ...... & $\begin{array}{l}\text { Rivulus cylindraceus... } \ldots \ldots \ldots \ldots \ldots \\
\text { Aplocheilus latipes. } \ldots \ldots \ldots \ldots \ldots \ldots \ldots \\
\text { Panchax panchax } \ldots \ldots \ldots \ldots \ldots \ldots \ldots \\
\text { Aphyosemion australis } \ldots \ldots \ldots \ldots \ldots \ldots\end{array}$ & $\begin{array}{r}13 \\
2 \\
8 \\
9\end{array}$ \\
\hline CyPRINIDAE $\ldots \ldots \ldots \ldots$ & 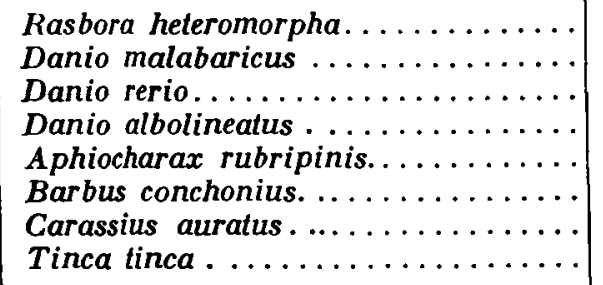 & $\begin{array}{r}6 \\
12 \\
2 \\
2 \\
6 \\
6 \\
5 \\
1\end{array}$ \\
\hline Poecilidan $\ldots \ldots \ldots \ldots$ & $\begin{array}{l}\text { Xiphophorus helleri } \ldots \ldots \ldots \ldots \ldots \ldots \\
\text { Lebistes reticulatus } \ldots \ldots \ldots \ldots \ldots \ldots \ldots \\
\text { Platypoecilus maculatus. ... } \ldots \ldots \ldots\end{array}$ & $\begin{array}{r}11 \\
3 \\
10\end{array}$ \\
\hline Melanotaenidae. ...... & Melanotaenia nigrans... . . . . . . . & $7 *$ \\
\hline Cichlidae. ....... & $\begin{array}{l}\text { Hemichromis bimaculatus. } \ldots \ldots \ldots \ldots \\
\text { Pterophyllum scalare } \ldots \ldots \ldots \ldots \ldots\end{array}$ & $\begin{array}{l}1 * \\
3\end{array}$ \\
\hline Centratchidae. $\ldots \ldots \ldots$ & Eupomotis gibbosus. ........... & 3 \\
\hline
\end{tabular}


Après cet aperçu sur la fréquence des cas de tuberculose observés plus spécialement sur poissons exotiques vivant en aquarium, on peut se demander comment il se fait que les cas de tuberculose pisciaire spontanée soient excessivement rares dans la nature.

Trois raisons peuvent être invoquées pour expliquer cette rareté :

$1^{0}$ la tuberculose se manifeste chez toutes les espèces animales surtout dès que les individus sont mis en groupes compacts, en espace restreint. Ce groupement des individus favorise la contagion et favorise également l'observation et le dépistage de l'affection;

$2^{\circ}$ dans la nature, les poissons atteints sont éparpillés dans le vaste milieu aquatique; beaucoup d'entre eux, en état de déficience, deviennent la proie facile soit de leurs congénères, soit d'animaux ichtyophages;

$3^{\circ}$ depuis les recherches de Bartarelli et Bocchia, personne n'a recherché systématiquement les lésions tuberculeuses (qui ne sont pas toujours facilement visibles) sur les poissons pêchés, soit au moment de la pêche, soit sur les marchés, soit dans les usines de conserves. Les autopsies systématiques déprécient la marchandise, et les sondages au hasard ne sont d'aucune valeur statistique.

La dernière question qui se pose, est celle de savoir si la tuberculose des poissons est contagieuse à l'homme et quel rapport peut exister entre les bacilles de la tuberculose pisciaire et le bacille de la tuberculose humaine. Je répondrai de suite que la tuberculose pisciaire telle que je l'ai rencontrée ne peut être contagieuse à l'homme, la raison majeure étant l'impossibilité (peut-être provisoire) des bacilles acido-résistants isolés de cultiver à la température de $37^{\circ}$. Inoculés aux animaux de laboratoires (cobaye, lapin, poule) ils n'ont donné aucune lésion. Ces bacilles ne semblent pas être des bacilles tuberculeux humains, bovins ou aviaires modifiés et adaptés aux poissons. Ce ne sont pas non plus des bacilles para-tuberculeux, puisqu'ils sont pathogènes et provoquent chez les poissons une affection caractéristique parfaitement superposable à celle des vertébrés supérieurs, les bacilles paratuberculeux étant, en principe, des bacilles acido-résistants saprophytes. Peut-être forment-ils la transition entre les mycobactéries saprophytes et les mycobactéries pathogènes; peut-être faut-il voir en eux les ancêtres des bacilles de la tuberculose ; peut-être trouvera-t-on des souches établissant une filiation, évolution parallèle à celle des vertébrés. En attendant, nous ne pouvons que les placer derrière les mycobactéries pathogènes déjà connues, à savoir :

Mycobacterium tuberculosis var. hominis (tuberculose humaine) ;

bovis (tuberculose bovine);

muris (tuberculose du campagnol).

Mycobacterium avium (tuberculose des oiseaux).

Myc. paratuberculosis (agent de l'entérite hypertrophiante des bovidés).

Myc. leprea (agent de la lèpre humaine).

Myc. leprea murium (agent de la lèpre des rats).

Myc. piscium de Dubard et Bataillon (tuberculose des Carpes). 
Myc. marinum d'Aronson (tuberculose des poissons de mer).

Myc. ranae (tuberculose de la Grenouille).

Myc. thamnospheos d'Aronson (tuberculose des Serpents).

Myc. /riedmanii de Friedman (tuberculose de la Tortue).

Myc. anabanti du Macropode, 3 souches i Ces souches étant celles Myc. sp, 11 souches

que j'ai isolées.

Vraisemblablement, quelques-unes de ces souches pourront être identifiées à certaines de ces cinq dernières espèces, et plus particulièrement à Mycobactorium piscium. 